\title{
Role of FDG PET-CT in Distinction of Benign Thrombus and Tumor Thrombus in Oncological Patients
}

\author{
Pelin Ozcan Kara ${ }^{1 *}$, Emel Yaman Sezer ${ }^{2}$, Zehra Pinar Koc ${ }^{1}$, Eda Bengi Yilmaz ${ }^{3}$ and Elvan Caglar Citak \\ ${ }^{1}$ Department of Nuclear Medicine, Mersin University, Turkey \\ ${ }^{2}$ Department of Oncology, Mersin University, Turkey \\ ${ }^{3}$ Department of Radiation Oncology, Mersin University, Turkey \\ ${ }^{4}$ Department of Pediatric Oncology Mersin University, Turkey \\ Received: February 17, 2018; Published: February 22, 2018 \\ *Corresponding author: Pelin Ozcan Kara, Mersin University Hospital, Nuclear Medicine Dpt. 33343 Mersin/Turkey, Tel: 903242410000; Fax: 903242410098; \\ Email: ppelinozcan@gmail.com
}

Abstract

Aim: Accurate diagnosis of tumor thrombus and distinguishing benign thrombus from tumor thrombus avoid unnecessary anticoagulant treatment of oncological patients and it is important for patient management. In this retrospective study, we aimed to demonstrate the role of FDG-PET/CT imaging for distinction of benign thrombus from tumor thrombus.

Materials and Methods: FDG-PET/CT imaging performed for staging, restaging and treatment response in oncological patients with thrombus were evaluated for accurate diagnosis of tumor thrombus and distinguishing benign thrombus from tumor thrombus by the examination of maximum standard uptake value (SUVmax). PET-CT imaging were evaluated both with visual assessment and increased metabolic activity. PET-CT findings were confirmed by clinical, radiological follow-up and biopsy in some cases.

Results: PET-CT imaging findings of a total of 37 patients with suspected tumor thrombus ( 25 men, 12 women, mean age: 53.62 -range: 8-82) were evaluated, retrospectively. Tumor thrombus in 11 segments in 8 patients ( 9 linear, 2 focal) and benign thrombus in 45 regions in the other 29 patients were reported in the evaluation of a total of 56 thrombi region in 37 patients on visual analysis and according to the increased metabolic activity. The most common region of thrombosis was defined as vena cava inferior. SUVmax values ranged from 7.91 to 20 (mean: 12.8) in 8 tumor thrombus patients. Compared to mediastinal region increased metabolic activity was not detected in none of the 29 patients with benign thrombus and these patients were treated with anticoagulant agents.

Conclusion: As a conclusion, FDG PET-CT imaging may be useful in oncological patients when radiological imaging findings are suspicious for tumor thrombus.

Keywords: FDG; PET-CT; Tumor Thrombus

Abbreviations: VTE: Venous Thromboembolism; TT: Tumor Thrombosis; US: Ultra Sonography; CECT: Contrast-Enhanced Computed Tomography; MR: Magnetic Resonance Imaging; FDG: Fluoro-De-Oxyglucose; PET-CT: Positron Emission Tomography/Computed Tomography

\section{Introduction}

Thrombosis because of venous thromboembolism (VTE) or tumor thrombosis (TT) is generally more common in oncological patients compared with nononcological patients. Tumor thrombus is a very rare but serious complication of solid tumors. The correct distinction of VTE and TT in oncological patients is critically important for patient management. VTE is cured with anticoagulant treatment. Accurate diagnosis of tumor thrombus and distinguishing benign thrombus from tumor thrombus provides selection of appropriate therapy. Radiological imaging procedures such as ultrasonography (US), contrast-enhanced computed tomography (CECT), magnetic resonance imaging (MR) finds out thrombus insidentally. However, differantial diagnosis is mostly difficult with these modalities. Fluorodeoxyglucose (FDG)Positron Emission Tomography/Computed Tomography (PET-CT) is a proven procedure in staging, restaging, diagnosis and treatment response in oncological patients.This retrospective study was designed to investigate the clinical role of PET/CT for accurate diagnosis of tumor thrombus and distinguishing benign thrombus 
from tumor thrombus to avoid unnecessary anticoagulant treatment of oncological patients.

\section{Materials and Methods}

\section{Patients}

We retrospectively reviewed all FDG PET/CT examinations between August 2014-April 2017 at Mersin University Hospital, and included all oncology-nononcology cases with FDG-avid tumor thrombosis and benign thrombus in the report. A total of 37 patients with tumor thrombus and benign thrombus were included. Informed concept was taken from all patients. PET-CT findings were confirmed by clinical-radiological follow-up at least six months and biopsy in some cases. Histopathology in one tumor thrombus patient and follow-up information after PET/CT scanning served as the standards of reference.

\section{Imaging Protocol}

All patients with blood glucose levels $<200 \mathrm{mg} / \mathrm{dl}$ and fasted for at least $6 \mathrm{~h}$ before an FDG injection of 370-555 MBq (10-15 $\mathrm{mCi})$. PET/CT scans were obtained $60 \mathrm{~min}$ after injection using an integrated scanner (GE Discovery PET/CT 610, US). A whole-body CT scan was performed without intravenous contrast administration with $130 \mathrm{kV}, 50 \mathrm{mAs}$, a pitch of 1.5 , a section thickness of $5 \mathrm{~mm}$, and a field of view of $70 \mathrm{~cm}$. A PET scan was performed immediately after an unenhanced CT scan, and acquired from the skull base to the upper thigh or foot with a 3-min acquisition per bed position using a three-dimensional acquisition mode. PET images were reconstructed iteratively by applying CT data for attenuation correction, and co-registrated images were interpreted.

\section{Image Analysis}

FDG-PET/CT imaging performed for staging, restaging and treatment response in oncological patients with thrombus were evaluated for accurate diagnosis of tumor thrombus and distinguishing benign thrombus from tumor thrombus by the examination of maximum standard uptake value (SUVmax). PET-CT images were evaluated both with visual assessment and increased metabolic activity in thrombus region. PET-CT images were evaluated both with visual assessment and increased metabolic activity by two experienced Nuclear Medicine physician. PET/CT images were viewed in the coronal, axial, and sagittal sections. The diagnosis of tumor trombus was based on FDG-avid solid masses inside the vessels in patients with known malignancy. The SUVmax was calculated. SUVmax of thrombus lesions were calculated on PET/CT by using region of interest (ROI) included at least twothirds of the lesions. Partial volume effect was minimized by this way. The regions were drawn by generating sphere circles. The quantitative uptake values of FDG (SUVmax) in the ROIs were semiautomatically calculated using workstations (GE).

\section{Statistical Analysis}

Statistical analysis was carried out with SPSS software (SPSS Inc., Chicago, Illinois, USA). A P value of less than 0.05 was considered statistically significant.

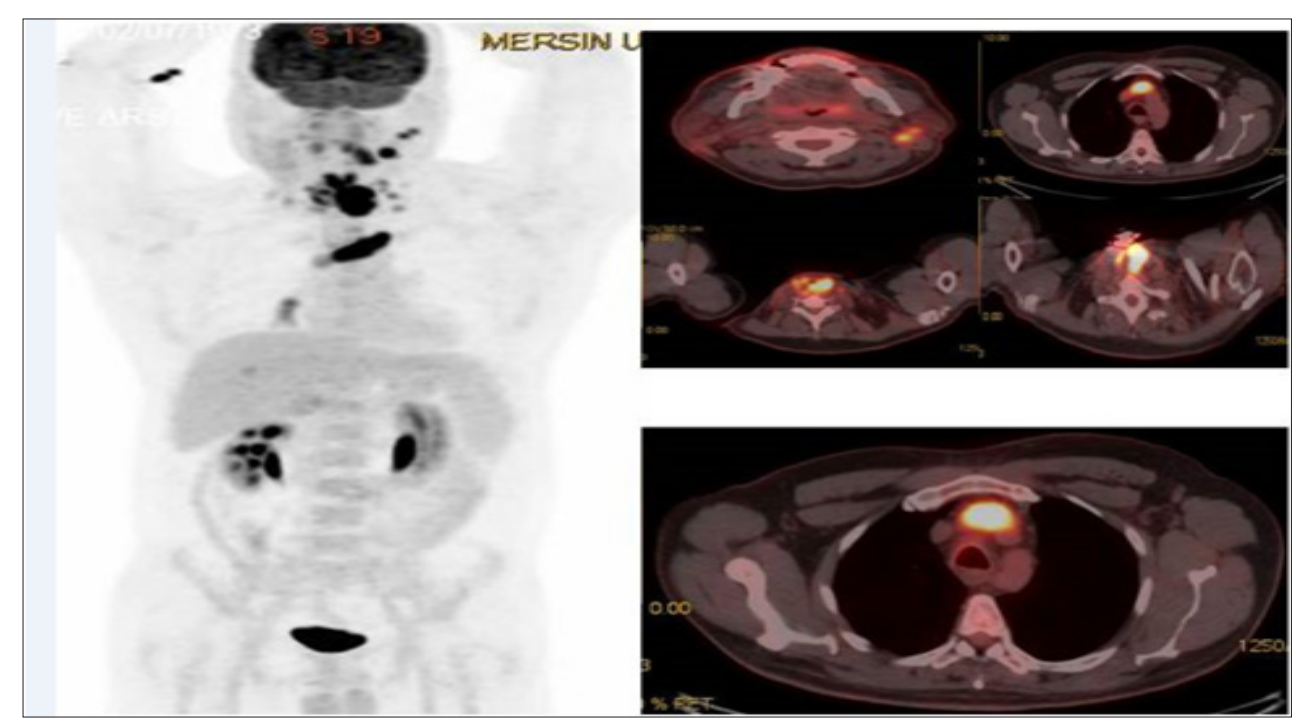

Figure 1: MIP (Maximum Intensity Projection) image and axial fusion images demontrate left brachiosephalic vein TT with SUVmax: 19.45 in a recurrent laringeal cancer 42 year old male patient.

\section{Results}

FDG-PET/CT imaging performed for staging, restaging and treatment response in oncological patients and patients with suspected malignancy. A total of 37 patients with tumor thrombus and benign thrombus were included. Patients who had confirmed primary malignancies ( lung cancer in 7 patients, gastrointestinal and hepatobiliary malignancies in 13 patients, genitourinary malignancies in 8 patient, sarcoma in 2 patients, lymphoma in 1 patient, laringeal cancer in 2 patients or suspected malignancies in 4 patients) were undergone PET-CT imaging. The most common malignancy was lung cancer (Table 1). PET-CT imaging findings of a total of 37 patients with suspected tumor thrombus (25 men, 12 women, mean age: 53.62-range: 8-82) were evaluated retrospectively. Of the 37 patients 56 thrombus regions ( 45 benign and 11 malignant thrombus) were shown by PET-CT. A total of 8 $(21.6 \%$ ) patients (7 M, $1 \mathrm{FM}$ ) with mean age 47.75 (Age Range: 
8-69) were included in malignant thrombus group (Group I). A total of $29(78.4 \%)$ patients (14 M, 7 FM) were included in benign thrombus group (Group II). Two patients had more than one vessel, one of which had 3 and the other had 2 malign thrombus regions in group I. FDG uptake pattern was linear in 9 of the 11 segments and focal in 2 segments. (Figures 1-3) illustrates three demonstrative tumour thrombus patients' PET-CT images. Tumor thrombus in 11 segments in 8 patients and benign thrombus in 45 regions in the other 29 patients were reported in the evaluation of a total of 56 thrombi region in 37 patients on visual analysis and according to the increased metabolic activity. Various vessels were affected. The most common region of thrombosis was defined as vena cava inferior. SUVmax values ranged from 7.91 to 20 (mean:12.8) in 8 tumor thrombus patients. Compared to mediastinal region increased metabolic activity was not detected in none of the 29 patients with benign thrombus and these patients were treated with anticoagulant agents.

Table 1: Suspected malignancy.

\begin{tabular}{|c|c|}
\hline Primary lesion & No. of patients \\
\hline Lung cancer & 7 \\
\hline Gastrointestinal and & 13 \\
\hline hepatobiliary malignancies & 2 \\
\hline Sarcoma & 8 \\
\hline Geitourinary malignancies & 2 \\
\hline Laringeal & 1 \\
\hline Lymphoma & 4 \\
\hline Suspected malignancy & \\
\hline
\end{tabular}

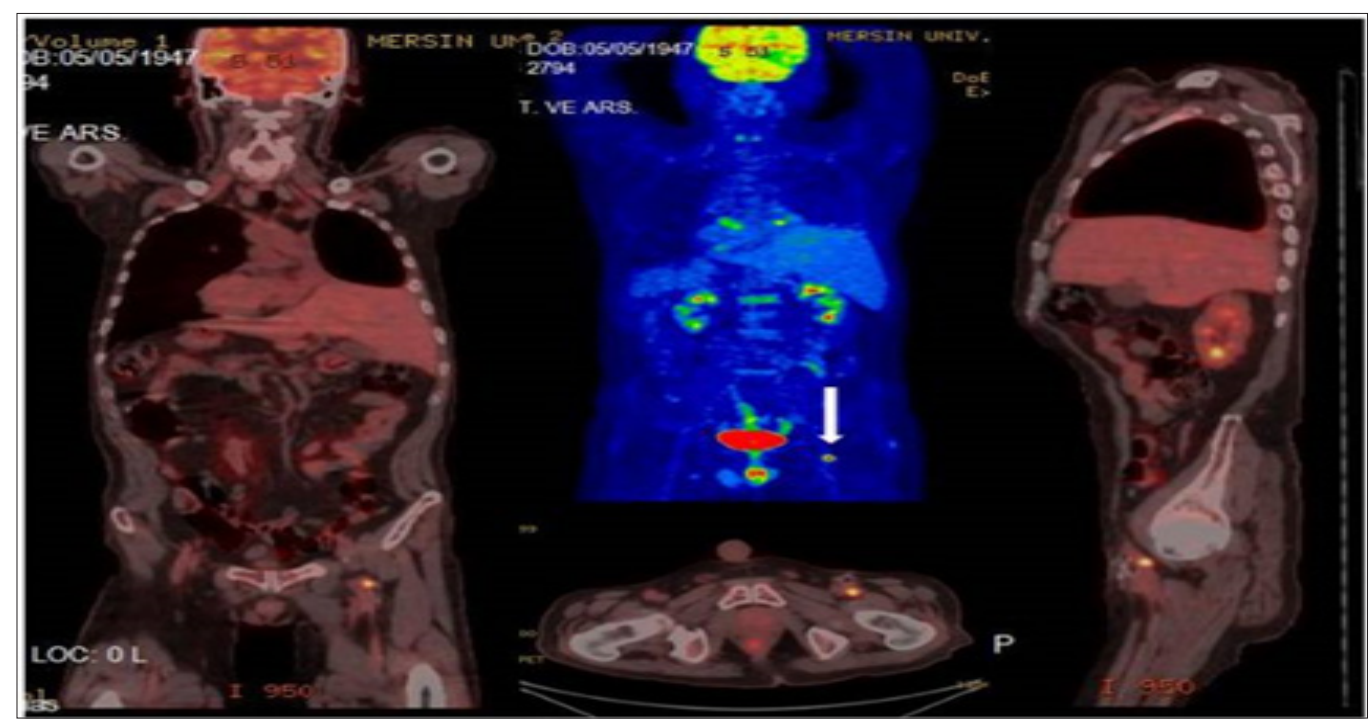

Figure 2: Left main femoral artery (white arrow) biopsy proven focal TT (SUVmax:9.27) in a lung cancer 68 year old male patient.

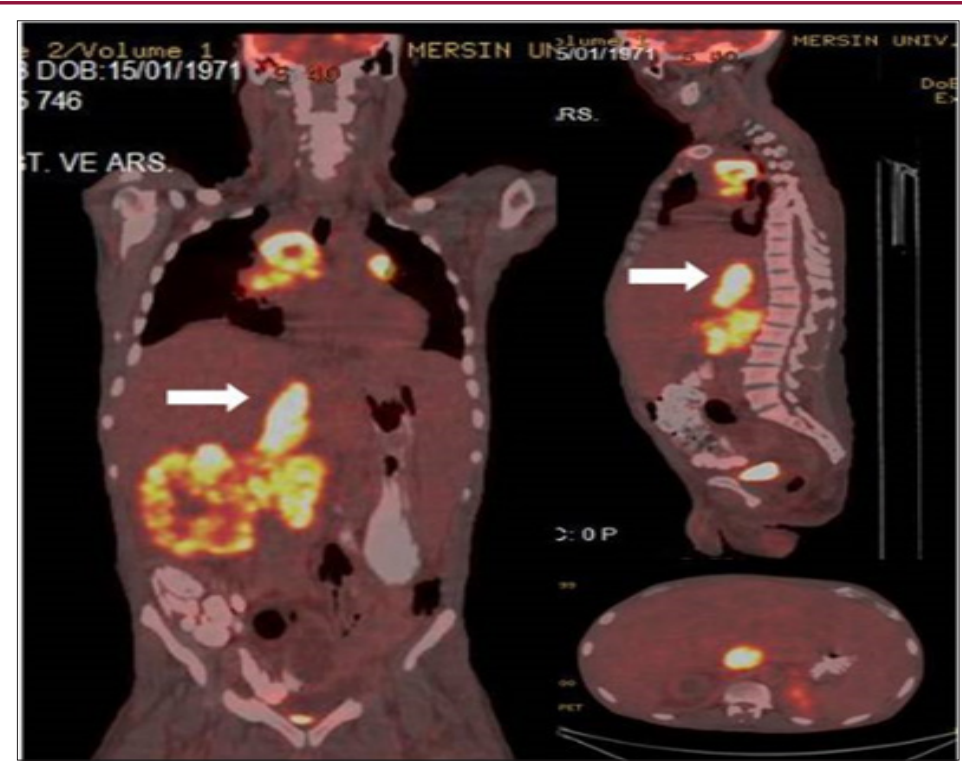

Figure 3: Coronal, sagittal and axial fusion images demontrate dilated VCI linear TT (SUVmax 16.88) with primary right RCC and widespread metastases in a 45 year old male patient. 


\section{Discussion}

Differentiation of malignant tumor thrombus from benign VTE lesions is a critical issue in clinical practice due to the different threatment strategies. Conventional imaging modalities such as US, venography, CECT, and MRI are used routinely in such cases. However, differantial diagnosis is mostly difficult with these modalities. TT is mostly an insidental finding in CECT. Tumor thrombus is rarely seen in solid tumors such as mostly in hepatocellular carcinoma (ca) and renal cell ca, followed by ovarian/testicular tumors, colon ca, pancreas ca, lung, breast, thyroid tumors, lymphomas, sarcomas, neuroendocrine tumors [1-16]. Maximum standardized uptake value (SUVmax) is semiquantitative parameter that reflect metabolic activity, but is not specific marker of malignancies. However, high FDG accumulation in a thrombus generally indiates a tumor thrombus. In a recent interesting image report from Sonavane SN et al, the authors reported the imaging findings of a patient with RCC where PET/CT not only ruled out locoregional adenopathy and distant metastases, but also distinguished tumor thrombi from benign thrombi in the same patient [16].

In a similar study in the literature for distinguishing benign and malignant tumor thrombus reported $71.4 \%$ sensitivity and specificity values of $\% 90$ with SUVmax cut-off value of 3.63 , while in another study cut-off value has been reported as 2.25 [14-16]. A recent study of 12 patients on the efficacy of FDG PET/CT for assessing the acuity of DVT found similar results with regards to BT with mean SUVmax of 2.41 [17]. FDG uptake pattern is not well established in TT. In the current study, we observed that both focal and mostly linear uptake pattern can be seen in TT. Our results are in line with literature with FDG uptake patern was linear in 9 of the 11 segments and focal in 2 segments. According to authors from a study by Ravina M et al. [18], these differences probably reflect variations in the intrathrombic density of neoplastic cells. The most common region of thrombosis was defined as vena cava inferior in line with literature. SUVmax values ranged from 7.91 to 20 (mean:12.8) in TT which was higher than that of VTE. SUV or Tumor/Liver SUV ratios per se are semiquantitative parameters that reflect metabolic activity, but are not specific markers of malignancies. Dual-time-point imaging (DTPI), which employs both early (first hour) and delayed scans (second hour or later) have been introduced to overcome the nonspesificity of PET. DTPI is acquisition of 2 PET scan one of which after $60 \mathrm{~min}$. and second after 120-180 min. following FDG injection.

Malignant cells have upregulated GLUT transporter and hexokinase activity, trapping FDG $[19,20]$. If a malignant cell is present, the continued FDG uptake between early and late scans results in higher intensity of retained FDG. Inflammatory cells, which retain normal glucose-6-phosphatase activity, will have decreased signal. The different pattern of FDG uptake in malignant versus nonmalignant cells is lower glucose-6-phosphatase levels in cancerous tissue. Inflamatuar and infectious processes have different FDG uptake pattern because of higher level of glucose-6phosphatase. As the uptake of 18F-FDG in malignancies is expected to increase over time DTPI could be potentially useful in partially overcoming the relatively low specificity of the SUVmax value.

\section{Conclusion}

The current study supports results from other small series regarding the usefulness of FDG PET-CT imaging in differentiation of TT from VTE. All linear and focal FDG uptake patterns with high SUVmax values were consistent with TT. Dual-time-point imaging as potential imaging protocol in differentiation of malignant lesions from benign lesions in larger series may be searched in further studies. As a conclusion, FDG PET-CT imaging may be useful in oncological patients with insidentally detected thrombus by conventional imaging or when radiological imaging findings are suspicious for tumor thrombus.

\section{References}

1. Lai P, Bomanji JB, Mahmood S, Nagabhushan N, Syed R, et al. (2007) Detection of tumour thrombus by 18F-FDG-PET/CT imaging. Eur J Cancer Prev 16(1): 90-94.

2. Davidson T, Goitein O, Avigdor A, Zwas ST, Goshen E (2009) 18F-FDGPET/CT for the diagnosis of tumor thrombosis. Isr Med Assoc J 11(2): 69-73.

3. Gupta P, Kramer EL, Ponzo F (2005) FDG uptake in tumor thrombus in inferior vena cava from rectal cancer on positron emission tomography. Clin Nucl Med 30(5): 342-343.

4. Nguyen BD (2005) Pancreatic neuroendocrine tumor with portal vein tumor thrombus: PET demonstration. Clin Nucl Med 30(9): 628-629.

5. Kaida H, Ishibashi M, Kurata S, Uchida M, Hayabuchi N (2007) Tumor thrombus in the inferior vena cava from colon cancer detected by 18F-FDG-PET. Ann Nucl Med 21(3): 185-188.

6. Kurtovic J, Van Der Wall H, Riordan SM (2005) FDG PET for discrimination between tumor extension and blood thrombus as a cause for portal vein thrombosis in hepatocellular carcinoma: important role in exclusion of transplant candidacy. Clin Nucl Med 30(6): 408-410.

7. Tateishi U, Yamaguchi U, Terauchi T, Maeda T, Moriyama N, et al. (2005) Extraskeletal osteosarcoma: extensive tumor thrombus on fused PET-CT images. Ann Nucl Med 19(8): 729-732.

8. Strobel K, Steinert HC, Bhure U, Koma AY, Gassmann N, et al. (2007) Tumour thrombus in the superior vena cava from anaplastic carcinoma of the thyroid: FDG-PET/CT imaging findings. Eur J Nucl Med Mol Imaging 34: 813.

9. Aurangabadkar HU, Palle L, Ali Z (2013) Tumour thrombosis and patterns of fluorine-18 fluorodeoxyglucose uptake: a pictorial review. Nucl Med Commun 34(7): 627-637.

10. Peiro V, Alonso Farto JC, García Poza J, Alfonso JM (2013) Inferior mesenteric venous thrombosis and F-18 FDG PET/CT. Rev Esp Med Nucl Imagen Mol 32: 55-56.

11. Probst S, Seltzer A, Chachoua A, Friedman K (2010) Azygos venous tumour thrombus from renal cell carcinoma detected by F-18 FDG PET/ CT. Clin Nucl Med 35(10): 832-833.

12. Beadsmoore CJ, Cheow HK, Salaetal E (2005) HCC thrombus in paraumbilical vein: detection by F-18 FDG PET imaging. Br J Radiol 78: 841844.

13. Jacoby WT, Cohan RH, Baker ME, Leder RA, Nadel SN, et al. (1990) Ovarian vein thrombosis in oncology patients: CT detection and clinical significance. AJR Am J Roentgenol 155(2): 291-294.

14. Sharma P, Kumar R, Singh H, Jeph S, Patnecha M, et al. (2012) Imaging thrombus in cancer patients with FDG PET-CT. Jpn J Radiol 30(2): 95104.

15. Lee EY, Khong PL (2013) The value of 18F-FDG PET/contrast-enhanced CT in detection of tumor thrombus. Clin Nucl Med 38(2): e60-e65. 
16. Sonavane SN, Malhotra G, Asopa R, Upadhye T (2015) Role of fluorine-18 fluorodeoxyglucose positron emission tomography in a case of renal cell carcinoma to differentiate tumor thrombus from bland thrombus. Indian J Nucl Med 30(4): 355-357.

17. Rondina MT, Lam UT, Pendleton RC (2012) (18)F-FDG PET in the evaluation of acuity of deep vein thrombosis. Clin Nucl Med 37(12): 1139-1145.

18. Ravina M, Hess S, Chauhan MS, Jacob MJ, Alavi A (2014) Tumor thrombus: ancillary findings on FDG PET/CT in an oncologic population. Clin Nucl Med 39(9): 767-771.

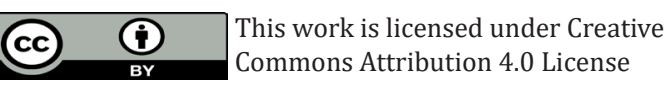

Submission Link: http://biomedres.us/submit-manuscript.php
19. Jones C, Badger SA, Lynch T, Diamond T (2010) Role of PET-CT in the management of colorectal metastatic disease. Oncol News 5(1): 17-19.

20. He YX, Guo QY (2008) Clinical applications and advances of positron emission tomography with fluorine-18-flurordeoxyglucose (18F-FDG) in the diagnosis of liver neoplasms. Postgrad Med J 84(991): 246-251.

$\begin{array}{ll}\text { BIOMEDICAL } & \text { Assets of Publishing with us } \\ \text { RESEARCHES } & \text { - Global archiving of articles } \\ & \text { - Immediate, unrestricted online access } \\ & \text { - Rigorous Peer Review Process } \\ & \text { - Authors Retain Copyrights } \\ \end{array}$

